\title{
Nicola Liebert \\ Schuldenkrise: Die Position der Gläubiger und das \\ Potential für einen Schuldenerlaß *
}

Zusammenfassung: Seit dem offenen Ausbruch der internationalen Schuldenkrise im Jahr 1982 und der damit verbundenen Gefährdung des internationalen Finanzsystems ist es den Banken gelungen, ihre ökonomische Position stets zu verbessern. Infolge des Aufbaus von Rücklagen, der Verlagerung ihrer Aktivitäten auf neue Märkte und Akteure und der Nutzung von Finanzinnovationen wie z.B. Debt EquitySwaps haben die Banken bis Ende der achtziger Jahre die verschuldeten Länder immer mehr in die Defensive drängen können. Was die Banken im internationalen Schuldenkonflikt gewonnen haben, haben die exportorientierten Unternehmen der entwickelten kapitalistischen Länder, insbesondere der USA, verloren. Die ökonomischenVoraussetzungenfür eine-wenigstens teilweise-Streichung der Schulden sind bei den Banken erfüllt. Die politische Durchsetzung eines solchen Schritts scheint dagegen in weiter Ferne.

Ruft man sich ins Gedächtnis, was die sogenannten Strategien zur Lösung der Schuldenkrise bislang erbracht haben, wird man zum Ergebnis gelangen, daß von einer Lösung der Krise nicht gesprochen werden kann: Der Schuldenberg wächst und wächst, mittlerweile auf ca. 1,2 Bio. US\$; die Verelendung in den Entwicklungsländern (EL) steigt im selben Maße. In Lateinamerika beispielweise ist im Durchschnitt aller Länder das pro-Kopf BSP von 1981 bis 85 um 13,2\% geschrumpft (berechnet nach Sachs 1986). Betrachtet man die derzeitige Situation näher, kommt ein zweiter Aspekt zum Vorschein: Zwar ist die Krise aus der Sicht der Schuldnerländer unverändert dramatisch, jedoch aus der Sicht der Gläubiger, der transnationalen Banken, ist das bisherige Schuldenmanagement durchaus als erfolgreich zu bezeichnen. Und tatsächlich sind die vorherrschenden Strategien (Umschuldungen, IWF-Anpassungsprogramme, unfreiwillige Kreditvergabe der Banken, Baker-Plan, Debt-Equity Swaps) allesamt in erster Linie nicht auf Problemlösung ausgerichtet ${ }^{1}$, sondern auf Systemstabilisierung. Die Banken, und damit das internationale Finanzsystem, sind nicht mehr unmittelbar bedroht durch renitente Schuldner. Ende der achtziger Jahre scheinen sie sogar wieder eine Position der Stärke erlangt zu haben. Darüber hinaus sind sie auch auf die EL als Kunden nicht mehr angewiesen, da sie ihre Geschäfte mittlerweile auf andere Märkte verlagert haben.

Nicht nur Banken gehören allerdings zu den Betroffenen, sondern auch die nichtfinanziellen Unternehmen und Regierungen der Gläubigerländer. Es hat sich gezeigt, daß die Interessen der Gläubiger nicht homogen sind: Das Finanzkapital steht gegen

Mein besonderer Dank gilt Klaus Enderle für seine vielfältigen Anregungen. 
das produktive Kapital. Bislang folgten die Krisenstrategien ausschließlich den Interessen des Finanzkapitals, der Banken - jetzt wird es sich, vor allem in den USA, nicht mehr auf Dauer abwenden lassen, daß auch die Interessen des produktiven Kapitals, und zwar sowohl des für den inländischen Markt als auch für den Export produzierenden Kapitals, stärker berücksichtigt werden. Genaus dies erscheint heute, da die Bankenposition gestärkt ist, um so mehr nötig und auch möglich. Diese Interessenstrukturen und deren Veränderung werde ich im dritten Abschnitt diskutieren.

Die aufgestellten Überlegungen führen dazu, eine Streichung der Schulden, zumindest eines Teils, als angemessene Strategie herauszustellen, was im vierten Abschnitt geschieht. So weit, so gut, wird man nun denken, da weiß man sich mittlerweile nicht nur mit Alfred Herrhausen, sondern auch mit der US-amerikanischen Regierung einig. Es soll daher bereits an dieser Stelle betont werden, daß die Überlegungen hier nicht enden dürfen, sondern daß ein Schuldenerlaß nur der allererste kleine Schritt auf dem Weg zu einer Lösung der grundlegenden Probleme der EL sein kann.

\section{Der finanzielle Sektor: Wie gefährdet ist das Finanzsystem?}

Nach der 'Eruption' der Krise im August 1982, als Mexico seine Zahlungsunfähigkeit bekannt geben mußte, stellte das »Nachbeben « eine starke Bedrohüng für das Finanzsystem der kapitalistischen Welt dar. Schnell wurden Rettungsmaßnahmen ergriffen: So erhielt Mexico Überbrückungskredite von der Bank für Internationalen Zahlungsausgleich (BIZ) und dem US-Finanzministerium, der IWF stellte Mittel aus der »Erweiterten Fonds Fazilität« zur Verfügung, die mexikanische Zentralbank konnte auf ihre Swap Line mit der Federal Reserve Bank of New York zurückgreifen, und die US-Regierung zahlte Vorschüsse auf zukünftige Ölimporte. Kurz darauf wurden ähnliche Maßnahmen für Brasilien und Argentinien notwendig. Durch sich anschließende Umschuldungen in Verbindung mit der Einschaltung des IWF wurde das Schuldenproblem zwar nicht gelöst, aber vertagt, so daß die Banken Zeit gewannen und sich bemühen konnten, die Situation zu entschärfen. Zunächst geschah dies durch weitere, 'unfreiwillige' Kreditvergabe an die Schuldner'; in anderen Worten heißt dies nichts anderes, als daß die Banken sich die Zinsen auf die EL-Kredite selbst bezahlten, um den Anschein, die Zahlungen seien einzubringen, aufrechtzuerhalten. Diese Kredite werden daher auch als 'recycling loans' bezeichnet, womit angedeutet wird, daß sie nicht für produktive Investitionen in den EL verwendet werden, sondern sogleich wieder an die Gläubiger in Form von Zinszahlungen zurïckfließen. Auch die Bankenaufsichten der Gläubigerländer standen den in Bedrängnis geratenen Geschäftsbanken zur Seite, indem sie es zuließen, daß auch eindeutig uneinbringbare Kredite zum vollen Nennwert in den Bilanzen belassen werden konnten, so daß Abschreibungen, die sich die Banken zunächst nicht hätten leisten können, nicht notwendig waren.

Die Schuldenkrise ist für die Banken zwar riskant, aber keineswegs ein ökonomisches Desaster. Zwar mögen viele Bankmanager in Anbetracht des Schuldengebirges 
schlechte Träume gehabt haben, zur selben Zeit aber konnten die Privatbanken exorbitante Gewinne realisieren. 1985 erhielten sie $30 \mathrm{Mrd}$. US\$ mehr aus den EL als sie an neuen Krediten an diese Länder vergaben (World Debt Tables 1987); im gleichen Jahr betrug der Gewinn etwa der US-amerikanischen Citicorp aus ihren Lateinamerikageschäften 250 Mio. US\$, was einem Viertel ihres Gesamtgewinns in jenem Jahr entspricht (Guardian 23.9.1987). Am Beispiel Brasiliens wurde gezeigt, daß die Gläubiger insgesamt gar den doppelten Betrag dessen einnehmen, was sie seit 1970 an das Land geliehen haben, wenn Brasilien in den nächsten 5 Jahren die fälligen Zinsen auf seine Kredite entrichtet (Altvater, Hübner, in dies. 1987). Den Banken gelang es also, sich durch anfänglich beträchtliche Gebühren für Umschuldungen und hohe spreads (Aufschläge) über den internationalen Zinssätzen LIBOR und Prime Rate für umgeschuldete Kredite schadlos zu halten. Dazu strichen sie zumindest einen Großteil der Zinsen für die EL-Kredite ein, wobei noch hinzuzufügen ist, daß die internationalen Zinssätze in der ersten Hälfte der 80er Jahre außerordentlich hoch waren ${ }^{3}$.

Dennoch war in Bankenkreisen nicht länger zu verleugnen, daß in der Schuldenfrage beide Seiten, Schuldner wie Gläubiger, in einem Abhängigkeitsverhältnis zueinander stehen - die Schuldnerländer gerieten in ökonomische und finanzielle Abhängigkeit, die Gläubiger aber sind von finanzieller Instabilität bedroht. Es setzte sich die Überlegung durch, daß die Banken, wenn es ihnen gelänge, sich von den Risiken der Schuldenmisere abzukoppeln, eine weitaus stärkere Verhandlungsposition erlangten und sich keine Sorgen mehr um unfreiwillige Kredite, andauernde Umschuldungsverhandlungen und störrische Schuldner zu machen brauchten. So weit wie möglich soll zwar der Schuldendienst gesichert werden, jedoch sind die Bilanzen von schlechten Risiken zu befreien. Die Abhängigkeit der EL könnte dagegen fast risikolos verewigt werden, denn diejenigen Verbindlichkeiten, die die Schuldnerländer weiterhin zu bedienen imstande sind, sollen bei den Banken verbleiben.

Die auf diesen Erwägungen aufbauende Strategie der Banken setzt sich aus vier Elementen zusammen:

1. Seit Ausbruch der Krise wurde die Neukreditvergabe an EL drastisch zurückgefahren, wie aus Tabelle 1 hervorgeht; von einem Höhepunkt von $52 \mathrm{Mrd}$. US\$1981, im Jahr vor dem Ausbruch der Krise, auf bloße 5 Mrd. US\$ im Jahre 1986. An die

Tabelle 1: Finanzflüsse von Geschäftsbanken an EL

(einschl. kurzfristige Kredite, in Mrd. US\$)

\begin{tabular}{l|rrrrrrr} 
& 1980 & 1981 & 1982 & 1983 & 1984 & 1985 & $1986^{*}$ \\
\hline $\begin{array}{l}\text { alle EL } \\
\text { Latein- }\end{array}$ & 49,0 & 52,0 & 37,6 & 34,1 & 17,4 & 13,5 & 5,0 \\
amerika & 36,0 & 41,0 & 31,5 & 15,1 & 14,0 & 5,0 & 1,0 \\
\hline
\end{tabular}

* geschätzt

Quelle: OECD, Financing and External Debt of Developing Countries, 1987 
lateinamerikanischen Schuldner wurden im selben Jahr gar nur noch 1 Mrd. US\$ Kredite von Geschäftsbanken vergeben.

Den Banken gelang es, ihre ausstehenden Forderungen an EL merklich zu vermindern, nicht nur indem sie die Kreditvergabe an EL fast völlig einstellten, sondern auch durch den Verkauf von Schuldtiteln (mehr davon unten) und inzwischen auch zunehmend durch Abschreibungen. Tabelle 2 stellt den Umfang von Forderungsreduktionen von US-Banken dar: Es zeigt sich, daß die Forderungen der US-Banken an EL insgesamt, die 1982 noch um 8,5\% angewachsen waren, sich 1984 erstmalig verringerten (um -1,8\%); 1986 waren es gar $-12,5 \%$, d.h. sie verminderten sich um die Summe von $17,3 \mathrm{Mrd}$. US\$. Im Falle der lateinamerikanischen Länder waren die Banken im Rahmen von Umschuldungen häufiger zu der Vergabe von neuen Krediten gezwungen, so daß hier eine weniger deutliche Verminderung der ausstehenden Forderungen zu verzeichnen ist, jedoch ist mit einer zeitlichen Verzögerung die gleiche Tendenz erkennbar wie bei den EL in ihrer Gesamtheit. Auf die Gründe, warum die größten US-Banken, wie aus der Tabelle ersichtlich, nicht ganz so gut dastehen, werde ich noch ausführlicher eingehen. Es wird in jedem Fall deutlich, daß die Banken seit 1984 durchweg (dies gilt auch für Banken anderer Gläubigerländer) eine zunehmende Verbesserung ihrer Position erreichen konnten.

Tabelle 2: Veränderungen von ausstehenden Forderungen von US-Banken in Mrd. US \$ und in \%

\begin{tabular}{|c|c|c|c|c|c|c|c|c|c|c|}
\hline & \multicolumn{2}{|c|}{1982} & \multicolumn{2}{|c|}{1983} & \multicolumn{2}{|c|}{1984} & \multicolumn{2}{|c|}{1985} & \multicolumn{2}{|c|}{$1986^{*}$} \\
\hline & Mrd. \$ & $\%$ & Mrd. \$ & $\%$ & Mrd. \$ & $\%$ & Mrd. \$ & $\%$ & Mrd. \$ & $\%$ \\
\hline $\begin{array}{l}\text { Forderungen an } \\
\text { kapitalimpor- } \\
\text { tierende EL }\end{array}$ & & & & & & & & & & \\
\hline alle US-Banken & 11,6 & 8,5 & 5,7 & 3,9 & $-2,7$ & $-1,8$ & $-11,9$ & $-7,9$ & $-17,3$ & $-12,5$ \\
\hline 9 größte & 7,3 & 8,7 & 3,3 & 3,6 & $-0,8$ & $-0,8$ & $-6,7$ & $-7,1$ & $-8,8$ & $-10,1$ \\
\hline $\begin{array}{l}\text { Forderungen an } \\
\text { Lateinamerika }\end{array}$ & & & & & & & & & & \\
\hline alle US-Banken & 6,9 & 8,2 & 2,1 & 2,3 & 1,8 & 1,9 & $-4,5$ & $-4,7$ & $-6,9$ & $-7,5$ \\
\hline 9 größte & 4,1 & 8,5 & 1,1 & 2,1 & 2,5 & 4,7 & $-1,5$ & $-2,7$ & $-2,6$ & $-4,8$ \\
\hline
\end{tabular}

* basierend auf Daten des ersten Halbjahres umgerechnet auf ein Jahr

Quelle: Watson, Kincaid u.a. 1986

2. Für die verbleibenden Verbindlichkeiten begannen die US-Geschäftsbanken ab Mai 1987, Rückstellungen ${ }^{4}$ vorzunehmen. Citicorp ging voran mit 3 Mrd. US\$, Chase Manhattan (1,6 Mrd. US\$), Bank America (1,1 Mrd. US\$), Manafacturers Hanover (1,7 Mrd. US\$) und andere folgten (Guardian, 21.8.1987). Zum Vergleich: Das Engagement dieser Banken in den EL betrug zur gleichen Zeit bei Citicorp 11,6 Mrd. 
US\$, Chase 7,0 Mrd. US\$, Bank America 7,3 Mrd. US\$, Manufacturers 7,6 Mrd. US\$ (siehe Tabelle 3). Den US-Banken folgten die britischen Banken; die deutschen und schweizer Banken hatten bereits vor längerer Zeit Rücklagen in Höhe von durchschnittlich ca. 30\% ihrer ausstehenden Kredite an EL anzusammeln begonnen. Die japanischen Banken zogen sich besonders elegant aus der Affäre: Sie gründeten ein Konsortium aus 28 führenden japanischen Banken, das Kredite an hochverschuldete Länder übernimmt und entweder Wertberichtigungen vornimmt oder die Kredite weiterverkauft - die Verluste der neuen Gesellschaft werden durch steuerpolitische Erleichterungen erträglich gemacht, und die Bilanzen der Banken sind von 'faulen' Krediten gesäubert (Wall Street Journal, 18.3.1987). Tabelle 3 zeigt, daß die Rückstellungen der großen US-Banken im Mai 1987 zwischen 13\% und 29\% ihrer ausstehenden Lateinamerika-Kredite betrugen, bei westdeutschen Banken gab es Rückstellungen von $50 \%$ bzw. sogar $70 \%$ bezogen auf Kredite an EL allgemein.

Tabelle 3: Rückstellungen der größten Privatbanken, Mai 1987

Ausstehende Kredite in Mrd. US \$
Rückstellungen in \% der ausstehenden Kredite

\begin{tabular}{l|rl}
\hline US-Banken* & & \\
Citicorp & 11,6 & 25 \\
Bank America & 7,3 & 29 \\
Chase Manhattan & 7,0 & 15 \\
Morgan Guaranty Trust & 4,6 & 20 \\
Chemical & 5,3 & 20 \\
Manufacturers Hanover & 7,6 & 13 \\
Westdeutsche Banken** & & \\
Deutsche Bank & 3,4 & 70 \\
Dresdener Bank & 3,4 & 50 \\
\hline
\end{tabular}

* Kredite an lateinamerikanische Länder

** Kredite an $31 \mathrm{EL}$

Quelle: Economist, 30.5 .1987

Es kann den Banken durchaus als große Leistung angerechnet werden, wenn die BIZ schätzt, daß von 1982 bis 1985 sich durch Erhöhung von Eigenkapital und Rücklagen der internationalen Banken der Zehnergruppe das Verhältnis von ausstehenden Forderungen zu Kapital um mehr als 25\% vermindert hat (BIZ 1987). Die großen Regionalbanken der USA (z.B. Security Pacific aus Los Angeles oder Continental Illinois aus Chicago) haben im Laufe des Jahres 1987 gar Rückstellungen von bis zu $50 \%$ ihrer ausstehenden EL-Kredite vorgenommen. Dadurch und durch das Vorpre- 
schen einiger finanzstarker Money-Center-Banken (insbesondere Citicorp) waren diverse Großbanken schon fast an die Wand gestellt, da sie nicht mitzuziehen in der Lage waren ${ }^{5}$. Für eine Zeitlang sah es sogar so aus, als wäre die Einigkeit der Banken untereinander, durch die sie die Macht eines Gläubigerkartells erlangt hatten, durch den Konkurrenzkampf gefährdet. Die Gefahr scheint aber nunmehr gebannt: Im Januar 1988 erklärten die stärksten New Yorker Money-Center-Banken (Bankers Trust und J.P. Morgan), keine weiteren Rückstellungen mehr vornehmen zu wollen. Dies bewirkte ein Aufatmen in den Führungsetagen manch anderer Banken - für Manufacturers Hanover insbesondere hätten Rückstellungen von etwa $50 \%$, wären sie nötig geworden, das Auslöschen ihrer gesamten Kapitalbasis bedeutet (Economist, 31.1.1988). Zwar birgt das Konkurrenzverhältnis der Banken untereinander immer ein Risiko für die Eintracht der Gläubiger in sich, jedoch hat es sich jetzt herausgestellt, daß es den Banken gelungen ist, den Schuldnerländern gegenüber an Stärke zu gewinnen und sich durch Rückstellungen abzusichern, ohne dabei diese Position der Stärke durch übermäßige Konkurrenzkämpfe zu gefährden. Rückstellungen bestärken die Banken nun noch darin, keine Kredite mehr an EL zu vergeben, können sie es doch nicht vor ihren Aktionären und Einlegern rechtfertigen, mit einer Hand Kapital zurückzulegen und so auf das Risiko der ausstehenden Kredite hinzuweisen und gleichzeitig mit der anderen Hand neue Kredite an dieselben Risikoschuldner zu vergeben.

3. Ohnehin war in den letzten Jahren eine Abkehr von traditionellen Kreditgeschäften zu verzeichnen. Durch Finanzinnovationen ${ }^{6}$ erschlossen sich die Banken die Möglichkeit, ihr Portfolio zu diversifizieren und einen Ausgleich zu schaffen für ihre durch uneinbringliche EL-Schulden belasteten Aktivgeschäfte. Allgemein handelt es sich hier um die Emission von handelbaren Wertpapieren ('securitization'), also die Schaffung von fiktivem Kapital, statt Kreditvergabe. Durch die Ausweitung dieses Anleihenmarktes, der fast ausschließlich auf Geschäfte innerhalb der OECD ausgerichtet ist, schaffen sich die Banken eine zunehmende Unabhängigkeit von den EL als ihren wichtigsten Geschäftspartnern der 70er Jahre. Betrug beispielsweise der Anteil der Neukreditgeschäfte der Banken mit EL 1982 noch 27\%, so war es im Jahre 1986 nur mehr 1\% (Watson, Kincaid u.a. 1986). Die EL sind nun nicht nur von den traditionellen Krediten abgeschnitten, sie sind auch fast vollkommen aus den neuen Finanzmärkten ausgeschlossen. Waren die Banken in den 70er Jahren noch von den EL als Kunden abhängig ${ }^{7}$, ist es ihnen jetzt gelungen, trotz nach wie vor bestehender Überliquidität in den kapitalistischen Industrieländern (IL) einen Markt von ungeahnter Größe zu erschließen, der ohne die riskanten Geschäfte mit der Peripherie auskommt ${ }^{8}$.

4. Gerade innerhalb des letzten Jahres rückte eine weitere Art von neuen Finanzgeschäften in den Mittelpunkt der Aufmerksamkeit: Schuldenswaps und in erster Linie dabei Debt-Equity Swaps. Diese Swaps sind zwar nicht neu. Bereits kurz nach dem Ausbruch der Krise 1982 kam es zu den ersten Schuldenaustauschaktionen zwischen Banken, aber erst die qualitativ neue Methode der Debt-Equity Swaps rückte diese Transaktionen in das allgemeine Interesse. Gehandelt werden die Schuldtitel auf 
Sekundärmärkten, und zwar mit einem Abschlag, der das Risiko widerspiegelt, das diese unsicheren Kredite darstellen. Mittlerweile sind die Marktwerte der Schulden zahlreicher Länder unter die 50\%-Marke bezogen auf den Nennwert gefallen (vgl. Financial Times, 2.10.1987).

Die Swaps der Banken untereinander können verschiedene Ausprägungen annehmen. So können zum einen Schuldtitel von verschiedenen Schuldnerländern getauscht werden (loan swaps), und zwar entweder Titel zu gleichen Nominalwerten, bei denen dann die Differenz der Marktwerte durch zusätzliche Zahlungen ausgeglichen werden (par swaps), oder Titel zu gleichen Marktwerten (ratio swaps), zum anderen können Forderungen mit unterschiedlichen Verzinsungsarten (etwa festverzinslich/variabel oder LIBOR/Prime Rate) getauscht werden (interest swaps); zum dritten werden in unterschiedliche Währungen denominierte Schulden getauscht (currency swaps). Swaps kommen dem Interesse der Banken entgegen, ihr Portfolio so weit wie möglich zu diversifizieren, um von einem etwaigen Moratorium oder einer Zahlungsunfähigkeit einzelner Länder weniger gefährdet zu sein (Risikostreuung), wobei den loan swaps besondere Wichtigkeit zukommt. Auch ein Rückzug aus bestimmten Ländern oder Regionen kann anstrebenswert sein; so versuchten beispielsweise US-Banken verständlicherweise ihr Engagement in der Golf-Region zu verringern und tauschten aus diesem Grund mit arabischen Banken saudiarabische gegen lateinamerikanische Schulden.

Besonders kleinere Banken, die nur verhältnismäßig geringe Forderungen an einzelne Länder haben, versuchen, sich gänzlich von den Schulden einiger oder günstigstenfalls aller ihrer Schuldner zu befreien, indem sie Schuldtitel gegen Cash auf dem Sekundärmarkt verkaufen. Dabei nehmen sie zwar Verluste in der Höhe des Abschlags vom Nominalwert, mit dem die Forderungen auf dem Sekundärmarkt gehandelt werden, hin, dafür aber haben sie die Gewißheit, wenigstens einen Teil des verliehenen Geldes zurückzuerhalten, und sie befreien sich von dem Ärger ständiger Umschuldungen und unfreiwilliger Kreditvergabe - die erwähnten Verluste sind kaum mehr dramatisch zu nennen, nachdem die Banken Rückstellungen vorgenommen haben. Nur einen Haken gibt es hierbei: Seit die Banken durch die Rückstellungen eingestanden haben, daß die Kredite womöglich 'faul' sind (»Durch Citicorps Rückstellungen ergeht es der Welt auf einmal wie dem kleinen Jungen, der sah, daß der Kaiser gar keine Kleider anhat « [Guardian, 21.5.1987, Übers. N.L.]), fiel auch der Wert der Schulden auf dem Sekundärmarkt erheblich, so daß die Banken sich einem Teufelskreis gegenüber sehen - wegen der Abschläge brauchen sie Rückstellungen, dadurch vergrößern sich aber die Abschläge, sie brauchen weitere Rückstellungen usw. ${ }^{9}$. Eine weitere Einschränkung ist, daß strikt marktlogisch der Marktwert desto tiefer fällt, je mehr Schulden auf dem Sekundärmarkt angeboten werden; die Nachfrage für solch risikoreiche Papiere ist zu gering, und dasselbe gilt auch für die im folgenden beschriebenen Debt-Equity Swaps. Es ist absehbar, daß die Preise noch weitaus niedriger werden, sobald die japanischen Banken, die bislang noch nicht als Anbieter auftreten, wie angekündigt auf den Schuldenmarkt drängen. Für die Großbanken gibt es noch einen anderen Grund, sich nicht zu sehr im Sekundärmarkt für 
EL-Schulden zu engagieren: Sie sorgen sich um den Wert der verbleibenden, nicht 'geswapten' Schulden, denn wenn die Banken Abschläge vom Nominalwert der Schulden gewähren, kommt das einem Eingeständnis gleich, daß der Nennwert eben nicht mehr dem tatsächlichen Wert entspricht. Letztendlich könnte die Bankenaufsicht eine Wertberichtigung in den Bilanzen der Geschäftsbanken erzwingen, während die Schuldnerländer insistieren könnten, ihren Schuldendienst lediglich auf den Marktwert ihrer Schulden berechnet zu entrichten. Dies aber wären viele Banken zu verkraften nicht in der Lage. Daher halten sich die Großbanken hierbei zurück; sie verdienen lediglich daran, daß sie als Maklerinnen für Swaps kleinerer Banken fungieren. Generell vermeiden es die Banken, die Geschäfte auf dem Sekundärmarkt zu publik zu machen. Auch fürchten die großen Banken eine Schwächung ihrer Position bei Umschuldungsverhandlungen, wenn sie trotz hoher Abschläge noch auf einen Schuldendienst für den Nennwert der Schulden beharren.

Tabelle 4: Grundkapital (einschl. Anleihen und Rücklagen)

und Aktivvermögen der US-Banken

\begin{tabular}{|c|c|c|c|c|c|}
\hline & 1982 & 1983 & 1984 & 1985 & $1986 *$ \\
\hline Forderungen an EL in Mrd. US \$ & 140,0 & 143,9 & 140,7 & 131,7 & 124,6 \\
\hline $\begin{array}{l}\text { Verhältnis v. Forderungen an EL } \\
\text { zu gesamtem Aktivvermögen in } \%\end{array}$ & 11,1 & 10,8 & 10,0 & 8,6 & 8,1 \\
\hline $\begin{array}{l}\text { Verhältnis von Grundkapital zu } \\
\text { Forderungen an EL in \% }\end{array}$ & 50,4 & 55,1 & 65,5 & 80,0 & 88,8 \\
\hline
\end{tabular}

* 1. Hälfte

Quelle: Watson, Kincaid u.a. 1986

Hieraus ist zu folgern, daß Swaps nicht, wie vielfach verkündet, eine Lösung der Schuldenkrise darstellen. Vielmehr müssen Swap-Transaktionen im Zusammenhang mit der Strategie der Banken, ihr eigenes Problem der finanziellen Verwundbarkeit zu beheben, gesehen werden. In Verbindung mit den drei bereits genannten Maßnahmen ergibt sich, daß die Banken mittlerweile sich aus einer akuten Gefahrensituation befreit haben: Die Bankenforderungen an EL sind zwischen 1982 und 86 von 140 Mrd. US\$ auf 125 Mrd. US\$ gesunken, das Verhältnis von austehenden EL-Krediten $\mathrm{zu}$ dem gesamten Aktivvermögen der Banken fiel im selben Zeitraum von 11\% auf $8 \%$, während das Deckungsverhältnis von Grundkapital der Banken zu EL-Schulden sich von $50 \%$ auf $89 \%$ verbesserte (s. Tabelle 4 ). Es ist bezeichnend für die neue Stärke der Banken gegenüber den Schuldnerländern, daß sie sich beispielsweise durch das Schuldendienstmoratorium Brasiliens 1987 nicht einmal einzuschüchtern lassen brauchten. So erklärte etwa Citicorp ausdrücklich, daß die Bank sich nicht von Brasilien erpressen lassen wolle (Handelsblatt, 17.3.1987) - und tatsächlich erreichte 
Brasilien mit seinem Konfrontationskurs keine Verbesserung seiner Lage. Wenn, wie abzusehen ist, der derzeitige Trend weiter anhält, werden sich die Banken so weit von der Verschuldung der EL abkoppeln, daß zwar die Krise der EL nicht gelöst sein wird, wohl aber die Krise der Banken.

\section{Debt-Equity Swaps}

Für Dept-Equity Swaps gilt im wesentlichen das oben Gesagte. Auch hier verkaufen Banken Forderungen mit einem Abschlag, aber die Käufer verwenden diese nicht für spekulative Zwecke (high risk - high yield), sondern benutzen die Schuldtitel, um sie sich im Schuldnerland zum Nennwert in heimische Währung umtauschen zu lassen und dann mit diesem Geld Investitionen im Schuldnerland vorzunehmen. Des öfteren benutzen Banken selbst ihre Forderungen, um damit Investitionen in den Finanzsektoren der Schuldnerländer zu finanzieren, d.h. Beteiligungen an Banken und Versicherungen zu erwerben. Eine andere Möglichkeit, die allerdings nicht in allen Schuldnerländern zugelassen wird, ist, daß Bürger des Schuldnerlandes ihre ins Ausland verbrachten Devisen (Fluchtkapital) so mit Gewinn repatriieren. Der häufigste Fall ist jedoch, daß ein multinationales Unternehmen Schuldtitel aufkauft und damit entweder eine Neuinvestition tätigt bzw. einen bereits existierenden Betrieb erweitert oder - und das dürfte die am weitesten verbreitete Swap-Art sein - bestehende Unternehmen des Schuldnerlandes aufkauft oder zumindest eine Kapitalbeteiligung (equity) erwirbt. So ergibt das böse Wort vom Ausverkauf der Schuldnerländer Sinn. Im Gegensatz zu den oben genannten Arten von Swaps, die keine direkten Auswirkungen auf die Situation der jeweiligen Schuldnerländer haben, ergeben sich durch Debt-Equity Swaps nicht bloß Probleme für Banken, sondern in viel größerem Umfang für die Schuldnerländer. Der Vorteil für diese Länder, nämlich daß ein Teil ihres Schuldenberges abgetragen wird, wird mehr als aufgehoben durch die sich ergebenden Nachteile:

a) der Abschlag vom Nennwert kommt den Konzernen der kapitalistischen Industrieländer (IL), nicht den EL zugute;

b) viele der Investitionen hätten ohnehin stattgefunden, ohne daß das Schuldnerland dafür heimische Währung den Investoren zur Verfügung hätte stellen müssen;

c) Unternehmen, die bereits in dem betreffenden Schuldnerland tätig sind, könnten versucht sein, ihr Kapital von dort abzuziehen, bloß um es dann anschließend wieder mit Gewinn im selben Land zu reinvestieren;

d) die Liquiditätsbeschaffung im Schuldnerland für die Rückzahlung der Schulden in heimischer Währung an die Investoren, die nun im Besitz der Forderungen sind, birgt eine nicht zu unterschätzende Inflationsgefahr in sich, die nur durch eine Begrenzung des Swap-Volumens und vor allem durch eine Senkung der öffentlichen Ausgaben, Abbau von sozialen Leistungen, Rückgang der öffentlichen Investitionen usw. abzuwenden ist;

e) in der Konsequenz wird der Handlungsspielraum der Regierungen der EL einge- 
schränkt, da keine Finanzierungsmöglichkeit mehr für staatliches Handeln besteht;

f) zur Inflationsvermeidung müssen die inländischen Zinsen hochgehalten werden, wodurch Investitionen von Inländern benachteiligt werden;

g) die Kontrolle von nationalen Industrien geht in die Hände von Ausländern über;

h) zu guter Letzt ist zu betonen, daß der Kapitaltransfer aus den EL nicht aufhört durch die Umwandlung der Schulden, denn ausländische Investitionen müssen genauso wie Kredite mit Devisen bedient werden - die Outflows (Gewinntransfer und Kapitalrepatriierung) können auf längere Sicht sogar leicht den Schuldendienst, der auf die ursprünglichen Schulden hätte geleistet werden müssen, übersteigen ${ }^{10}$.

Aus all diesen Gründen versuchen die meisten EL, das Volumen der Swap-Transaktionen zu begrenzen; gleichzeitig halten sich, wie erwähnt, viele Banken zurück, und die Investoren ihrerseits klagen über mangelnde Anlagemöglichkeiten in den EL. Der Börsencrash vom Oktober 1987 hat potentielle Investoren gewiß nicht ermutigt, Kapitalbeteiligungen in EL zu erwerben, da nun Liquidität solch immerhin risikoreicher Anlagen vorgezogen werden dürfte. Der Markt ist daher kaum erweiterungsfähig. Die geschätzten Angaben über das Volumen der Swaps liegen für 1986 zwischen 5 Mrd. US\$ (Schubert 1987) und 6 Mrd. US\$ (Hannon/Haugen 1987), - eine äußerst unscheinbare Summe gegenüber einer Schuldenlast von mittlerweile etwa 1,2 Billionen Dollar. Debt-Equity Swaps werden also sicher nicht das Ende der Schuldenkrise einleiten; betrachtet man außerdem die zögerliche Haltung der Investoren und die verhältnismäßig geringe Zahl von profitablen Unternehmen, die in den EL zur Übernahme zur Verfügung stehen, und die oben beschriebenen Sorgen der Banken, ist es ebenso unsinnig anzunehmen, daß sich das ganze Streben der Gläubiger darauf richtet, »durch Umschuldungen Zeit zu gewinnen und während dieser Zeit ein möglichst hohes Volumen an Direktinvestitionen vor allem im Zuge der Debt-toEquity-Swaps durchzusetzen«, wie H. Schui dies vermutet (Schui 1988, S. 160). Stattdessen müssen Debt-Equity Swaps im Kontext der Bankenstrategie, die maximale Flexibilisierung und Unabhängigkeit der Banken anstrebt, gesehen werden, und hier erfüllen sie zweifellos ihre Aufgabe: Die Banken stehen stärker da denn je zuvor seit Ausbruch der Schuldenkrise.

\section{Der reale Sektor: Auswirkungen der Schuldenkrise auf die Wirtschaft der Industrieländer}

Die Schuldenkrise ist zweifellos ein Problem für Gläubiger und Schuldner - aber sind sie wirklich die einzig Betroffenen? Gibt es nicht auch andere Betroffene, deren Interessen nicht denen der Banken entsprechen und die deshalb andere Strategien favorisieren würden als jene? Im folgenden sollen diese Fragen am Beispiel der US-Wirtschaft beantwortet werden. Die USA stehen hier im Mittelpunkt der Betrachtung, da sie als Hegemonialmacht der kapitalistischen Welt und darüber hinaus als größter 
Gläubiger zumindest der lateinamerikanischen, also der am höchsten verschuldeten Länder ${ }^{11}$, bei der Formulierung von Lösungsstrategien immer die zentrale Rolle spielten und voraussichtlich auch weiter spielen werden. Viele der nun folgenden Argumente lassen sich jedoch auch auf andere westliche IL übertragen. Worum es sich hierbei handelt, wurde von A. Watkins prägnant zusammengefaßt:

»Die Schuldenkrise schadet der Gesundheit der US-Wirtschaft, der Durchführung der US-Außenpolitik, der Stabilität des Finanzsystems der USA und der Arbeitslosenrate in den USA - ganz zu schweigen von unserer nationalen Sicherheit ... Gegenwärtig wird die Politik der USA gegenüber der Schuldenkrise so formuliert, als seien die Banker die Einzigen, die ein besonderes Interesse an den Ergebnissen dieser Politik haben.« (Watkins 1986, S. VIII, Übers. N.L.)

Die Interessen der Vereinigen Staaten mit Bezug auf die Schuldenkrise sind durchaus widersprüchlich - die Verschuldung der EL bringt sowohl Vor-als auch Nachteile für die USA mit sich. So ist es zu erklären, daß die US-Strategien gegenüber der Schuldenkrise nie einheitlich und zielgerichtet waren. Die Vorteile, die die Verschuldung der EL für die Vereinigten Staaten und andere westliche IL mit sich brachte, sollen nur kurz angerissen werden. Zunächst einmal können aufgrund der Krise der EL Ressourcen in großem Umfang aus diesen Ländern abgezogen werden. Die Rohstoffimporte der IL verbilligen sich erheblich, seit sich die terms of trade der EL rasant verschlechtern ${ }^{12}$, wodurch unter anderem zu erklären ist, daß die Inflationsraten der IL ausgesprochen niedrig sind und die Monetaristen, die allerorten an der Regierung sind, scheinbare Erfolge vorweisen können. Es kommt neben diesem Realtransfer auch zu einem finanziellen Transfer aus den EL, da diese einerseits kaum noch Kapital aus den IL erhalten, andererseits aber hohe Schuldendienstzahlungen an die IL abführen. So bessern perverserweise die EL die Zahlungsbilanzen der IL auf, und zwar inzwischen immerhin mit fast 60 Mrd. US\$ jährlich (Economist, 26.9. 1987). Daß zu diesem doppelten Transfer nun auch die Möglichkeit hinzugekommen ist, zu Schleuderpreisen die Industrien der Schuldnerländer aufzukaufen, macht die Lage für die IL nur noch angenehmer. Es ist noch zu erwähnen, daß die IL durch die prekäre und abhängige Situation der EL einen politischen und ökonomischen Hebel gegen jene erlangt haben und sie mit Hilfe von IWF und Weltbank zu den IL genehmen wirtschaftlichen und politischen Reformen zwingen. Aus den genannten Faktoren erklärt es sich, warum die IL im Grunde gar kein besonderes Interesse hatten, die Schuldenkrise tatsächlich zu lösen. Umschuldungen boten sich an, um die EL in Verschuldung und Abhängigkeit zu halten, ohne das internationale Bankensystem kollabieren zu lassen. An diesen Vorteilen knüpft Schui (1988) mit seiner Interpretation der Schuldenkrise an. Ihm zufolge liegt dem ganzen Schuldendebakel eine groß angelegte, von langer Hand geplante Strategie der USA zugrunde, wobei er den Ausgangspunkt Ende der 60er Jahre ansetzt, als die Hegemonie der USA ins Wanken geriet und die Vorteilsposition des Dollars nicht mehr gewährleistet war. Spätestens 1979 werde dann eine neue US-Politik erkennbar, deren Grundideen darin bestehen sollen, daß durch eine hohe Sockelarbeitslosigkeit die Macht der Gewerkschaften gebrochen wird, gleichzeitig im Inland kleine und schwächere Kapitale ausgeschaltet werden, so daß sich auch bei stagnierender Nachfrage die dominierenden Unternehmen weiter vergrö- 
Bern können, und daß die Staatsnachfrage gezielt in den von diesen Unternehmen besetzten Wirtschaftszweigen (hauptsächlich Rüstung und high tech) erhöht wird. Im internationalen Bereich will diese Politik laut Schui den Kapitalexport rentabel machen und schwächere Kapitale weltweit ausschalten, um die Möglichkeiten für Direktinvestitionen zu verbessern. Die Methode, dies zu erreichen, basierte auf der Zinspolitik. Mit Absicht (!) soll demnach die US-Regierung ihre Hochzinspolitik verlängert und damit den Konjunkturabschwung ab 1980 vertieft haben. Der Dollar steigt, die Auslandskonkurrenten auf dem Binnenmarkt werden erfolgreicher, und die Arbeitslosigkeit nimmt zu. Gleichzeitig bewirkt der durch die hohen Zinssätze gestiegene Schuldendienst der EL, daß einerseits die US-Banken ihre Gewinne ausweiten können, andererseits geraten die EL in eine Krise, so daß die lokalen Unternehmen ruiniert werden und zur Übernahme durch IL-Konzerne bereitstehen. Letztendlich haben die USA nun die Möglichkeit, mit Hilfe von IWF und Weltbank die Wirtschaften der EL durch strikte Auflagen so umzuwandeln, wie es in das Interesse des USKapitals paßt. Angesichts von so viel Voraussicht kann es dann kaum noch verwundern, daß Schui bereits die Ölkrisen als Teil der US-Strategie sieht. Sind dann die neuen Strukturen, die Modernisierung und Spezialisierung der US-Industrie, geschaffen, kann die Konsolidierung beginnen, indem der Dollarkurs wieder gesenkt wird, so daß nun die US-Defizite beseitigt werden können.

In der Tat ist Schui darin zuzustimmen, daß die Schuldenkrise für bestimmte Interessengruppen erhebliche Vorteile mit sich bringt und auch, daß gewisse Interessen die Krise für sich funktionalisieren und die US-Politik gegenüber der Verschuldungskrise in ihrem Sinne beeinflussen konnten. Was aber an Schuis allzu logisch erscheinender Darstellung auffällt, ist, daß es sich um eine reine ex-post-Betrachtung handelt, in die alle Entwicklungen auf dem Weltmarkt ebenso wie in der US-Ökonomie eingefügt werden, so daß am Ende alles wie ein geradezu teuflisch zu nennender Plan aussieht. Nur hat er es sich etwas zu einfach gemacht: Ohne zu differenzieren bringt er die unterschiedlichsten Interessen und Akteure zusammen, nämlich Politiker, Finanzkapital, Gewerkschaften, binnenmarkt- und exportorientiertes Kapital. Der einzige Interessengegensatz, den er gelten lassen will, ist der zwischen zukunftsorientiertem 'big business' und kleinen Industrien mit rückständiger Technologie, wobei ersteres der Nutznießer der oben beschriebenen Strategie ist. Es erscheint jedoch unplausibel, daß sich Politiker so ausschließlich für die Interessen einer einzigen Kapitalgruppe einspannen lassen, zumal wenn die geforderte Strategie ständig so nahe am Rande des ökonomischen Abgrunds entlangmanövriert. Schui vergißt außerdem, daß es unmöglich im Interesse des Finanzkapitals gewesen sein kann, sich in eine Position zu begeben, in der es von dem good-will der EL abhängig war, den Schuldendienst weiter zu leisten, in der manche Banken zeitweise in ihrer Existenz bedroht waren und es Anlaß gab, um das gesamte Finanzsystem der kapitalistischen Welt zu fürchten. Umgekehrt kann es nicht im Interesse des produktiven Kapitals sein, daß nun die EL dazu gezwungen sind, ihre Importe drastisch zu kürzen und als Konkurrenten auf dem Weltmarkt ebenso wie auf dem Binnenmarkt der USA aufzutreten. Das multinationale Kapital wiederum hat kein Interesse daran, daß die EL zu tief in eine Krise ge- 
raten, denn je instabiler die ökonomischen Bedingungen in einem Land sind, desto weniger laden sie zu Direktinvestitionen in dem betreffenden Land ein. Wie der von Schui geschilderte komplizierte Plan, in dem angeblich mit Absicht wirtschaftlicher Abschwung und Krisen hervorgerufen wurden, politisch durchsetzbar gewesen sein soll, wo die politische Instanz, die solch weitgreifende und vorausschauende, aber gleichzeitig so risikoreiche Entscheidungen getroffen haben soll, ist, geht aus seinen Ausführungen nicht hervor. Auch bei nur geringer Kenntnis der politischen Strukturen der USA wird jedoch klar, daß es sich um ein extrem dezentralisiertes System handelt, in dem gerade die Interessenvielfalt und -gegensätzlichkeit politische Entscheidungen prägt, in dem Akteure, wie sie die von Schui formulierte Strategie voraussetzen würde, nicht existieren.

Es stellte sich im Laufe der Zeit heraus, daß die USA durch die Schuldenkrise eben nicht nur Vorteile haben, auch wenn die Fraktion des multinationalen high-techorientierten Kapitals sicherlich weniger von diesen Nachteilen betroffen war. Es ist einleuchtend, daß die Schuldnerländer erst genügend Exportgewinne realisieren müssen, um den Schuldendienst finanzieren zu können, d.h. derselbe Mechanismus, der den Banken ihre Gewinne ermöglicht, gereicht den Produzenten in den IL, zuvorderst in den USA, zum Nachteil, da die Produkte, die die Schuldner zu exportieren gezwungen sind, insbesondere auf den US-Markt drängen. Speziell im Zusammenhang mit dem US-Handelsbilanzdefizit ist dies gravierend. Je mehr Waren importiert werden, desto weniger werden im Land selbst produziert - aber geringere Produktion ist gleichbedeutend mit weniger verdientem Einkommen und damit auch mit geringeren Ausgaben, so daß der letztendliche Nachfrage- und Produktionsriickgang ein Vielfaches des ursprünglichen Handelsdefizits beträgt. Bislang gelang es den USA, die daraus resultierenden negativen Wachstumsimpulse durch rasant steigende Haushaltsdefizite zu kompensieren. Dies jedoch wird in Zukunft problematischer werden, da schon jetzt die Vereinigten Staaten die Defizite nur noch durch eine wachsende Auslandsverschuldung finanzieren können - das traditionelle Gläubigerland USA ist der Welt größter Schuldner geworden. Geringeres Wirtschaftswachstum jedoch bewirkt einen Rückgang der Importe, so daß die Chancen der EL, ihren Schuldendienst zu finanzieren, schrumpfen. Und dies wiederum führt zu Verlusten für die Banken.

Wie weit die Schuldenkrise mitverantwortlich ist für das US-Handelsbilanzdefizit, verdeutlichen folgende Zahlen: Etwa $85 \%$ der lateinamerikanischen Exportsteigerung gingen in die USA. Obwohl das lateinamerikanische Exportvolumen zwar im Verhältnis zur Größe des US-Marktes klein ist, hat es dennoch spürbare Auswirkungen, da die Exporte meist auf wenige Güter konzentriert sind. Was die Ausfuhren der USA betrifft, gingen $198141 \%$ in die EL, 1985 nur noch $34 \%$ (Economic Report of the President 1987). Der Rückgang der Exporte nach Lateinamerika von $37 \%$ im Zeitraum von 1981 bis 1983 ist für die Hälfte des gesamten Rückgangs der US-Exporte im selben Zeitraum verantwortlich (Lever 1984). Das bedeutet, die lateinamerikanischen Staaten, und nicht etwa Japan, waren die Hauptverursacher des enormen Anstiegs des US-Handelsbilanzdefizits. 1981 bis 1984 verschlechterte sich die Han- 
delsbilanz der USA mit Japan um etwas über 18 Mrd. US\$, mit Lateinamerika aber um 23 Mrd. US\$, von einem Überschuß von $7 \mathrm{Mrd}$. US\$ auf ein Defizit von $16 \mathrm{Mrd}$. US\$ (Watkins 1986). Außer auf die Handelsbilanz der Vereinigten Staaten kommt es auch zu Auswirkungen auf den Arbeitsmarkt: Schätzungen darüber, wieviele Arbeitsplätze durch die Schuldenkrise verlorengingen, schwanken zwischen 400000 und 2 Mio. (a.a.O.). Die Branchen, die besonders hart getroffen werden, sind ausgerechnet die Krisenbranchen der USA. Einerseits können gerade Produkte wie Maschinen, Fahrzeuge, Agrarprodukte, Stahl und ähnliches nur noch schlecht in den EL abgesetzt werden; so halbierten sich von 1980 bis 83 z.B. die Exporte von Fahrzeugen, Eisen und Stahl nach Lateinamerika (a.a.O.). Andererseits drängen viele Schuldnerländer ihrerseits mit ähnlichen Produkten auf den Weltmarkt (Brasilien beispielsweise mit Stahl, Argentinien mit Weizen und Sojabohnen) und verschlechtern so die Chancen der US-Anbieter. Besonderes dramatisch ist der Fall der Sojaexporte: 1980/81 kamen weltweit $40 \%$ aller Sojaexporte aus den USA ( 7200 t); die argentinischen Exporte waren mit 398 t unbedeutend. 1984/85 exportierten die USA noch 4700t, Argentinien dagegen 2400 t (a.a.O.). Die Farmkrise in den Vereinigten Staaten kann also nicht getrennt gesehen werden von der Verschuldungskrise der EL. Die USA, als bedeutender Rohstoffexporteur, leiden jetzt außerdem ihrerseits teilweise unter dem Rohstoffpreisverfall auf dem Weltmarkt. Auch politisch können sich den Interessen der USA zuwiderlaufende Probleme ergeben: Wird den EL nämlich eine immer drastischere Austerität aufgezwungen, sind sozialistisch orientierte Revolutionen oder fanatisch nationalistische Regierungen ein mögliches Ende.

Die Vereinigten Staaten stehen vor einem Dilemma: Um die Schuldenkrise wie bisher unter Kontrolle zu halten, um zumindest einen Teil des Schuldendienstes zu erhalten, müssen sie die notwendigen Dollars den Schuldnerländern zu Verfügung stellen. Da dies kaum noch über Neukredite geschieht, müssen die USA ein Handelsbilanzdefizit gegenüber den EL wohl oder übel hinnehmen, d.h. sie müssen den EL Exporte ermöglichen. Genau dies aber können sie sich derzeit nicht leisten. Finanzminister Baker hat das erkannt, als er seinen Plan 1985 formulierte, der den bis dahin üblichen Strategien widersprach und einen verstärkten Finanzfluß in die EL vorsah. Der BakerPlan aber war zum Scheitern verurteilt, da die Summen, von denen die Rede war (insgesamt knapp $30 \mathrm{Mrd}$. US\$), fast lächerlich zu nennen sind; aber noch nicht einmal die waren aufgebracht worden, da für die Banken nicht genügend Anreize gegeben waren, nach Bakers Wünschen 'dem schlechten Geld noch gutes hinterherzuwerfen'13. Baker hatte also denselben Fehler wie später Schui gemacht: er vernachlässigte die Interessengegensätze zwischen den verschiedenen Kapitalfraktionen innerhalb der USA. R. Dornbusch hat das Dilemma der USA folgendermaßen beschrieben:

»Wir stehen in der Tat unschlüssig vor einer Situation, wo wir uns entscheiden müssen. Wollen wir in die EL exportieren, dann müssen wir ihnen erlauben, mehr zu uns zu exportieren, um die Devisen zu verdienen, um für unsere Exporte und für ihre Schulden zu zahlen; oder wollen wir auf den Schuldendienst verzichten, dann verlieren wir keine Arbeitsplätze. Unsere Politik bislang war es, den Kopf in den Sand zu stecken und uns keine Sorgen um das Problem zu machen.« (in US Congress 1984, S. 43, Übers. N.L.) 


\section{Zwischenbilanz: Wie wäre es mit Schuldenerlaß?}

Würden allerdings die USA und andere Industrienationen zumindest einen Teil der Schulden erlassen, wäre das Dilemma schnell gelöst. Lange jedoch war das Thema Schuldenerlaß weitgehend tabu - inzwischen haben selbst Banker (Alfred Herrhausen und Henry Kaufman) und Politiker der Gläubigerländer (z.B. der britische Finanzminister Nigel Lawson) das Wort in den Mund genommen. Zum Jahreswechsel kam es sogar zu einer neuen Entwicklung an der Schuldenfront: Zwischen den USA und Mexiko wurde ein Abkommen geschlossen, in dem de facto die Streichung eines, wenn auch nur kleinen, Teils der Schulden vorgesehen war. Zunehmend wird erkannt, daß die Krise der EL auch den IL zum Schaden gereicht; die Eintreibung der Schulden ist nur noch um den Preis der doppelten Krise, nämlich in EL, die den Gürtel immer enger schnallen müssen, wie in den IL, wo Arbeitslosigkeit und - jedenfalls im Fall der USA - Handelsbilanzdefizite verschlimmert werden, möglich, und das lediglich zur Sicherstellung der Profite der transnationalen Banken. Heute müssen die EL durch die vom IWF verordneten Anpassungsmaßnahmen für die Aufbringung des Schuldendienstes $\gg$ Zwangssparen (Hankel 1984), also eine negative Einkommensund Entwicklungspolitik verfolgen und auf die Ausweitung ihrer Binnenökonomien verzichten, um etwas zu erreichen, was weder ihnen noch ihren Gläubigern nützt: exportorientiertes Wachstum und Importdrosselung - bloß damit den Banken Verluste erspart bleiben.

Bisher waren die rein an Bankeninteressen ausgerichteten Strategien immer dadurch gerechtfertigt worden, daß andernfalls das finanzielle Chaos drohe - und das wäre nicht nur für die IL, sondern auch für die EL katastrophal. Diese Argumentation hatte zweifellos ihre Berechtigung, jedoch sind die Finanzinstitute seit dem Ausbruch der Krise nicht auf der Stelle getreten. Genau dieser Punkt muß mehr Aufmerksamkeit finden. Die alten Argumente sind hinfällig geworden, denn wenn die Schuldenstrategien bislang einen Erfolg hatten, so war es der, das internationale Finanzsystem zu stabilisieren. Es wäre nun geradezu verwerflich, diesen Stabilisierungserfolg nicht zu nutzen und endlich auch die anderen von der Schuldenkrise betroffenen Akteure zum Zuge kommen zu lassen. Es muß Abschied genommen werden von der Vorstellung, ein Schuldenerlaß müßte in jedem Fall Verluste für die Gläubigerländer mit sich bringen, denn

»intemationale Finanzierung, einschließlich der EL-Schulden, ist kein Null-Summen-Spiel; ein Gewinn für einen Schuldner ist nicht automatisch ein Verlust für den Gläubiger.« (Decoodt 1986, S. 6, Übers. N.L.)

Was in den EL nicht für den Schuldendienst aufgewendet wird, wird für Konsum- und Kapitalgüter ausgegeben, die oftmals importiert werden, bzw. für eine Wachstumssteigerung, was wiederum zu mehr Importen führt. Ebenso wie also die Exportchancen der IL verbessert werden, vermindert sich die Exportkonkurrenz der EL, wenn diese nicht mehr zu Exportsteigerungen um jeden Preis gezwungen sind. Schuldenstreichung, so ergibt sich daraus, ist kein Transfer von der 'Ersten' in die 'Dritte Welt', sondern vielmehr ein Transfer innerhalb der 'Ersten Welt' vom Finanz- zum Indu- 
striesektor. Es ist hinzuzufügen, daß in den IL gerade die notleidenden Bereiche der Industrie unterstützt würden, da die EL-Importe zum Gutteil aus 'traditionellen' Industriegütern (Stahl, Fahrzeuge, auch landwirtschaftliche Produkte) bestehen.

Die Banken müßten sicherlich einige Verluste hinnehmen, was jedoch erträglich gestaltet werden kann, wenn nicht alle Schulden auf einen Schlag erlassen werden. Die Höhe der Verluste hängt von den Durchführungsmodalitäten ab, bei denen verschiedene Möglichkeiten bestehen: nur schrittweise eine Entschuldung vorzunehmen, überhaupt nur einen bestimmten Teil der Schulden zu erlassen oder nur bei den am schlimmsten betroffenen Ländern anzusetzen ${ }^{14}$. Einige Verluste würden nunmehr, wie dargestellt, die Banken auch nicht dem Untergang weihen. Ohnehin würden diese sich wahrscheinlich in Grenzen halten: So berechnet etwa C. Dziobek (1987), daß eine Abschreibung von $30 \%$ der Schulden und eine Streichung der Zinsen über LIBOR Verluste bedeuten würden, die nicht höher sind als die Gewinne der Banken im Zeitraum von 1983 bis 86 (!). Es muß bedacht werden, daß die Banken durch Debt-Equity Swaps und sonstige Verkäufe von Forderungen auf dem Sekundärmarkt bereits jetzt Verluste hinnehmen, d.h. also, daß sie offenbar unter bestimmten Bedingungen zu Abschreibungen bereit sind. Auch haben die Banken durchaus ihrerseits einen gewissen Anreiz, zumindest einen Teil der Schulden zu erlassen: Zeitraubende Umschuldungsverhandlungen und unfreiwillige Kreditvergabe werden überflüssig, und, was vielleicht wichtiger ist, im Vergleich zu einem Moratorium der Schuldnerländer hätte ein Schuldenerlaß den Vorzug, geplant und mit erheblich weniger Risiko durchgeführt werden zu können. Es besteht nämlich die Gefahr, daß die Schuldner, wenn die Banken sich durch die vier im zweiten Abschnitt dargestellten Strategien immer sicherer und unabhängiger von den EL fühlen, den Schuldendienst womöglich schlicht einstellen, denn dann ist der Anreiz, die Zahlungen aufrechtzuerhalten, nämlich die Hoffnung auf neue Kredite, nicht mehr gegeben.

\section{Schuldenerlaß konkret: der Fall Mexiko}

Das im Dezember 1987 geschlossene Abkommen zwischen den Vereinigten Staaten und Mexiko ${ }^{15}$ läßt mittlerweile hoffen, daß sich die im letzten Abschnitt dargestellten Überlegungen auch in den Köpfen der verantwortlichen Politiker und Banker durchsetzen. Längst dürfte (im Stillen) auch ihnen klargeworden sein, daß ein beträchtlicher Teil der Schulden ohnehin uneinbringlich geworden ist - die zunächst noch zaghafte Anerkennung der Realitäten war längst überfällig. Worum handelt es sich also? Der von Morgan Guaranty Trust für die Regierungen Mexikos und der USA ausgearbeitete Plan sah ursprünglich die Ausgabe einer sogenannten Null-KuponAnleihe, i.e. eine Anleihe, für die alle angefallenen Zinsen am Ende der Laufzeit auf einmal gezahlt werden, durch die US-Regierung vor, die mit allen anfallenden Zinsen und Zinseszinsen insgesamt $10 \mathrm{Mrd}$. US $\$$ wert sein sollte. Mexiko sollte dafür den Betrag von 2 Mrd. US $\$$ zahlen. Diese sollen zur Sicherheit in den USA hinterlegt werden. Mexiko soll daraufhin von den Gläubigerbanken ausstehende Forderungen 
zurückkaufen, und zwar wurde gehofft, zum Marktwert, der derzeit für mexikanische Verbindlichkeiten bei ca. 50 \% liegt. Die Banken erhalten dafür von Mexiko Bonds, die durch die US-Null-Kupon-Anleihe gedeckt sind; für die Zinszahlungen auf diese Papiere (1,6\% über LIBOR) ist weiterhin die mexikanische Regierung verantwortlich. Durch diesen Handel, so hoffte man, könnten somit etwa 20 Mrd. US\$ mit Hilfe der 10 Mrd. US\$ in Form von US-Anleihen zurückgekauft werden. Der Abschlag, den die Banken beim Verkauf ihrer Forderungen gewähren müssen, entspricht so einem Schuldenerlaß, da, anders als bei Debt-Equity Swaps, der Abschlag dem Schuldnerland selbst zugute kommt. Zum ersten Mal sieht also dieser Plan die Reduktion der Schuldenlast eines Landes vor, statt daß wie bei den bisherigen Strategien immer nur neue Schulden hinzugefügt werden.

Allerdings war klar, daß die Durchführung des Plans von den in Frage kommenden Banken abhing, deren Teilnahme freiwillig war. Zunächst begrüßten sie den Vorschlag: Die neuen Papiere bieten höhere Erträge und geringeres Risiko als die alten Forderungen; darüber hinaus können sie sich durch deren Erwerb von weiteren Kreditverpflichtungen befreien. Jedoch folgte prompt das Aber: Der Abschlag von $50 \%$ sei erheblich zu hoch. Der mexikanischen Regierung stand es jedoch frei, Gebote von Banken, die zu niedrig erschienen, auszuschlagen. Ende Februar 1988 fand die mit Spannung erwartete Versteigerung der neuen mexikanischen Bonds statt, und es zeigte sich tatsächlich, daß ein Abschlag von $50 \%$ bei weitem nicht zu realisieren war. $45 \%$ der abgegebenen Gebote wurden von Mexiko abgelehnt, so daß nur 3,7 Mrd. US\$ Schulden für einen durchschnittlichen Preis von $68 \%$ des Nennwerts, also im Austausch für neue Papiere im Wert von 2,6 Mrd. US\$, zurückgekauft werden konnten. Der Nutzen, den Mexiko damit durch dieses Geschäft hat, bleibt sehr begrenzt: Das Land spart in den nächsten 20 Jahren knapp über 2,6 Mrd. US\$ an Schuldendienstzahlungen, wobei $532 \mathrm{Mio}$. US für die US-Anleihe, durch die die neuen mexikanischen Bonds gedeckt werden, gezahlt werden müssen - dem stehen Schulden von insgesamt $103 \mathrm{Mrd}$. US\$ gegenüber (Financial Times, 4.3.1988). Quantitativ gesehen handelt es sich also höchstens um einen Tropfen auf dem heißen Stein. Jedoch sollte diese Maßnahme deswegen trotzdem nicht abgetan werden, denn qualitativ stellt sie eine neue Entwicklung in der Schuldenfrage dar. Als solche wollte sie auch das mexikanische Finanzministerium verstanden wissen, das nach Bekanntgabe des Ergebnisses der Versteigerung erklärte, die Aktion sei trotz des mageren finanziellen Gewinns ein Erfolg gewesen, da erstmalig die Gläubiger die Tatsache anerkannt haben, daß der Buchwert der Schulden nicht mehr den realen Wert widerspiegelt (a.a.O.) - dies könnte ein erster Schritt dahingehend sein, daß der Schuldendienst der EL in Zukunft nicht mehr auf den Nominalwert der Schulden, sondern auf den Marktwert berechnet geleistet zu werden braucht.

Warum die Banken sich nur so zurückhaltend an diesem Geschäft beteiligten, obwohl es in der Theorie nichts anderes bedeutet als das, was sie ohnehin schon häufig in Form von Verkäufen von Forderungen auf dem Sekundärmarkt bzw. Debt-Equity Swaps praktizieren, läßt sich durch mehrere Faktoren erklären: Die neuen mexikanischen Bonds sind zwar mit einem höheren Zinssatz versehen als es die alten Forderungen 
waren, aber obwohl sie durch die US-Anleihe gedeckt sind, stellen sie doch ein Risiko dar, da Mexiko nach wie vor für die Zinszahlungen verantwortlich ist; daher ist es wahrscheinlich, daß die Bonds ihrerseits bald auf dem Sekundärmarkt im Wert fallen. Die Banken müßten außerdem, wenn sie sich an dem Handel beteiligen, die Abschläge in einem großen Brocken verdauen der häufigeren kleinen Transaktionen an Stelle wie bisher üblich. Noch schwerwiegender ist, daB insbesondere viele von den Großbanken es vorziehen dürften, nicht durch ein solches Geschäft zuzugeben, daß die mexikanischen Verbindlichkeiten nur noch zwei Drittel oder die Hälfte ihres Nominalbetrags wert sind. Der Grund dafür ist, daß die Bankenaufsicht ihre Haltung zu der Frage, ob ein so offensichtliches Eingeständnis nicht dazu führen würde, daß die Banken dazu gezwungen werden, für die verbleibenden Forderungen entsprechende Wertberichtigungen vorzunehmen, noch nicht eindeutig geklärt hat. Immerhin hat die Wertpapier- und Börsenaufsichtsbehörde der USA den Banken im Fall einer Beteiligung an dem Tauschgeschäft bereits die buchhalterische Anerkennung von Verlusten auch für die im Portfolio der Banken verbleibenden Forderungen empfohlen (Frankfurter Rundschau, 29.2.1988). Abschreibungen in diesem Umfang auf einen Schlag zu verdauen, wäre für die meisten Banken bei dem derzeitigen Stand der Dinge inakzeptabel. Für kleinere Finanzinstitute mit geringen ausstehenden Forderungen an Mexiko, die sich aus diesem Land ganz zurückziehen wollen und dafür auch hohe Abschläge in Kauf zu nehmen bereit sind, ist der Erwerb der neuen Papiere sicher reizvoller. Aber auch hier gibt es Grenzen, ist es doch verhältnismäßig problemlos für diese Banken, auf dem Sekundärmarkt Cash für ihre Forderungen zu kaum geringeren Preisen zu erhalten; die neuen Bonds haben allerdings den Vorteil, wahrscheinlich leichter verkäuflich zu sein als die alten Schuldtitel. Es war also von vorneherein absehbar, daß die Großbanken sich zurückhalten würden und daß generell die Nachfrage und die gebotenen Abschläge gering sein würden.

Trotz dieser Einwände ist die Initiative eindeutig als begrußßenswert zu bezeichnen, da immerhin damit ein neuer Standard für künftige Verhandlungen zwischen Schuldnern und Gläubigern gesetzt wird. Von seiten der USA wurde allerdings sofort betont, daß ein solches Abkommen nur für Länder mit ausreichenden Devisenreserven, die sich den IWF-Programmen unterwerfen und die deutliche Anstrengungen zur Anpassung (nach US-Gusto) unternehmen, in Frage käme. Ob Brasilien, das kurz nach der Bekanntgabe des Abkommens mit Mexiko Interesse an einem vergleichbaren Handel äußerte, dies auch erreichen kann, sei deshalb dahingestellt. Da es sich aber bei dem Mexiko-Deal gezeigt hat, daß den Banken ein Schuldenerla 3 immer noch nicht schmackhaft genug gemacht werden konnte, wäre es wünschenswert, daß auf die Banken politischer Druck ausgeübt wird, einen Schuldenteilerlaß auch unter Hinnahme einiger Nachteile zu akzeptieren. Ein erster Schritt wäre es schon, wenn die Bankenaufsicht eine solche Maßnahme dadurch erleichtern würde, daß sie den Banken eindeutig zugesteht, ihre verbleibenden Forderungen zu den Buchwerten in den Bilanzen zu behalten. Es ist dennoch wichtig, den Mexiko-Plan in die Betrachtung aufzunehmen, zeigt er doch, daß Schuldenerlaß keine Utopie zu sein braucht, so nur der politische Wille vorhanden ist. Denn um ein politisches Problem handelt es sich 
hier schon längst: Politische Schritte sind gefordert, wo ökonomische Maßnahmen für die Verbesserung der Situation der Schuldner seit 1982 permanent versagt haben.

\section{Anmerkungen}

1 Als Lösungsstrategien beruhen sie sämtlich auf falschen Prämissen, z.B. daß eine drastische Ausfuhrsteigerung aus allen Entwicklungsländern auf einmal machbar sein soll, ohne daß die Rohstoffpreise weiter sinken und ohne daß der Protektionismus der Industrieländer eine Schranke bildet; oder daß rasantes Wachstum in EL wie in IL es den Schuldnerländern auf einmal ermöglicht, die zum wiederholten Mal umgeschuldeten Beträge irgendwann doch zurüickzuzahlen usw.

2 Die unfreiwillige Kreditvergabe war eine Bedingung, die der IWF zur Voraussetzung für seine Abkommen mit den Schuldnerländern machte. Da die Banken an vom IWF überwachten Anpassungsprogrammen in den EL sehr interessiert waren, mußten sie sich notgedrungen den dahingehenden Wünschen des IWF fügen. 1983 wurden im Zusammenhang mit Umschuldungen z.B. 13 Mrd. US\$ an neuen Krediten vergeben, 1984 waren es noch 10,4 Mrd. US\$ (World Debt Tables 1987).

3 Nominale und reale Zinssätze in \% Nominale Zinssätze
Libor US Prime Rate

Reale Zinssätze* Libor US Prime Rate LIBOR US Prime Rate

$\begin{array}{rrrrr}1980 & 14,0 & 15,3 & 0,6 & 1,7 \\ 1981 & 16,7 & 18,9 & 38,0 & 40,6 \\ 1982 & 13,6 & 14,9 & 33,7 & 35,2 \\ 1983 & 9,9 & 10,8 & 4,6 & 5,5 \\ 1984 & 11,3 & 12,0 & 10,0 & 10,7 \\ 1985 & 8,6 & 9,9 & 21,7 & 23,1\end{array}$

* deflationiert mit dem Rohstoffexportindex (außer Öl) aus den EL Quelle: UN World Economic Survey 1987

Es ist zu bedenken, daß die Kredite, die den EL in den 70er Jahren so großzügig zuflossen, mit variablen Zinssätzen versehen waren. D.h. die EL nahmen Kredite zu Niedrigstzinssätzen (real gesehen teilweise negativ) auf und fanden sich plötzlich in der Situation wieder, auf dieselben Kredite extreme Zinszahlungen leisten zu müssen.

4 Rückstellungen sind ein Passivposten in den buchhalterischen Bilanzen der Banken, die, anders als Rücklagen, die Gewinnrechnung belasten, d.h. den Gesamtgewinn schmälem. Der Vorteil gegenüber Rücklagen ist, daß die Banken so geringere Steuern zu zahlen haben. Rückstellungen oder Rücklagen sind notwendig, damit die Banken Wertberichtigungen vornehmen können. Dies bedeutet, daß die Bilanzen geändert werden, um den tatsächlichen Wert eines Aktivpostens (ausstehenden Kredits) wiederzuspiegeln; im Fall von Schuldtiteln ist das der Sekundärmarktwert. Bislang taten die Banken alles, um solche Wertberichtigungen zu vermeiden.

5 Daß aber ein Mitziehen bei weiteren Runden von Rückstellungen für die Banken zum Zwang wird, zeigt sich auch daran, daß der Aktienmarkt diejenigen Banken, die Rückstellungen oder Abschreibungen vornehmen, belohnt, während sich die verbleibenden Banken mit einem Sinken ihrer Aktienkurse konfrontiert sehen (Economist, 9.1. 1988).

6 Im einzelnen sollen hier aus Platzgründen die verschiedenen neuartigen Finanzierungsinstrumente nicht dargestellt werden. Es sei hier auf den Artikel von U. Traber (1986) verwiesen.

7 Wie sehr die Banken um die Gunst der EL buhlten, wird durch die scharfe Zinssatzkonkurrenz für ELKredite in den 70er Jahren verdeutlicht, so daß die Banken teilweise negative Realzinssätze hinnahmen (UN World Economic Survey 1987).

8 Dies wird vor allem dadurch möglich, daß die neuen Finanzierungsinstrumente auch gerade das durch die Überliquidität in den kapitalistischen IL im Überfluß vorhandene Spekulationskapital in die langfristige Finanzierung einbinden. 
9 Die Differenz zwischen Marktwert und Nennwert der ausstehenden Schulden war Anfang 1986 etwa genauso groß wie im Jahre 1987 die Differenz zwischen Marktwert und dem Niveau, bis zu dem im Durchschnitt Rückstellungen gemacht worden waren, da der Marktwert etwa im gleichen Maß fiel wie Rückstellungen vorgenommen wurden (Economist, 25.7. 1987).

10 Ausführlicheres zu den möglichen Problemen durch Debt-Equity Swaps findet sich in Hannon, Haugen (1987); BIZ (1987); Roberts, Remolona (1987).

111977 beispielsweise hielten US-Banken in 12 Ländem einen Anteil von $50 \%$ der Forderungen an die jeweiligen Länder (Nunnenkamp / Junge 1985).

12 Um die Devisen für den Schuldendienst aufbringen zu können, müssen die EL ihre Exporte - und das sind bei den meisten Schuldnerländern Rohstoffe - kräftig steigern. Tun aber alle Schuldner dasselbe, ist die unausweichliche Konsequenz, daß die Rohstoffpreise wegen des Überangebots auf dem Weltmarkt ins Rutschen kommen.

»Wenn der jährliche Schuldendienst nicht drastisch gekürzt wird ... und Rohstoffe nicht angemessen neubewertet werden, werden die lateinamerikanischen Länder und die Länder der Dritten Welt generell zu der fast vollkommenen Ausbeutung ihrer naturlichen Ressourcen verdammt sein ... Dies würde eine buchstäbliche territoriale Eroberung des Südens durch den Norden bedeuten, nicht durch einen offenbaren militärischen Konflikt, sondern im Namen der sakrosankten Konzepte 'Entwicklung' und 'Interdependenz'. (Schatan 1987, S. 23, Übers. N.L.)

13 Ausführlicheres zum Baker-Plan in BIZ 1985, Bogdanowicz-Bindert 1985 und 1986, Johnson 1985 u.a.

14 Zwei der bekanntesten Vorschläge, wie ein Schuldenerlaß gestaltet werden kann, stammen von W. Hankel (1983) und F. Castro (1985). Hankel plädiert für die Einrichtung eines Gegenwert-Fonds, in den der Gegenwert des Schuldendienstes in nationaler Währung des Schuldnerlandes eingezahlt werden müßte, so daß das Transfer-Problem gelöst wäre. Die Gelder aus dem Fonds, der gleichzeitig eine Art von internationaler Aufsicht darstellen soll, würden dann wiederum im Schuldnerland für Entwicklungsprojekte verwendet. Castro hingegegen unterscheidet zwischen legitimen und illegitimen Schulden (das sind diejenigen Schulden, die durch Entwicklungen außerhalb des Einflusses der EL entstanden, wie etwa durch Ölpreissteigerungen; dazu können auch die von Militärregierungen aufgenommenen Schulden zählen), wobei letztere nicht zurückzahlbar sein sollen. Jener Teil der Schulden soll von den Regierungen der IL übemommen werden und in Raten durch Abzweigungen vom Militärhaushalt abgetragen werden.

15 Warum gerade wieder Mexiko mit diesen neuen Maßnahmen bedacht wird, ist dadurch zu erklären, daß Mexiko wirtschaftlich das für die USA wichtigste Schuldnerland ist, es außerdem politisch gesehen direkt den 'Hinterhof' der USA darstellt und daß die USA sich nun, in Anbetracht von bevorstehenden harten Verhandlungen mit Brasilien und Argentinien, bei Mexiko den Rücken freihalten wollen.

\section{Literatur}

Altvater, E. / Hübner, K. u.a. (Hg.) (1987): Die Armut der Nationen. Handbuch zur Schuldenkrise von Argentinien bis Zaire, Berlin

Bank für Internationalen Zahlungsausgleich (1985): 55: Jahresbericht, Basel

Bank für Internationalen Zahlungsausgleich (1987): 57. Jahresbericht, Basel

Bogdanowicz-Bindert, C. (1985): World Debt - The United States reconsiders, in: Foreign Affairs, $64 / 2$

Bogdanowicz-Bindert, C. (1986): The Debt Crisis: The Baker Plan revisited, in: Journal of Interamerican Studies and World Affairs, 28/3

Castro, F. (1985): Die Verschuldungskrise der Weltwirtschaft und die aufhaltsame Zerstörung des Kapitalismus durch die Banken, in: Leviathan, 4

Decoodt, P. (1986): The Debt Crisis of the Third World: some aspects of causes und solutions, in: The Columbia Journal of World Business, 21/3, 1986 
Economic Report of the President (1987): together with the Annual Report of the Council of Economic Advisers, Washington, D.C.

Hankel, W. (1983): Die Finanzkrise zwischen Nord und Süd. Gründe, Lehren, Schlußfolgerungen, in: Simonis, U. (Hg.) (1983): Entwicklungsländer in der Finanzkrise. Probleme und Perspektiven, Berlin Hankel, W. (1984): Die Schuldenkrise, ein Problem der Dritten oder der Ersten Welt? Umschuldung oder Schuldenerlaß?, in: Blätter für deutsche und internationale Politik, 29/1, 1984

Hannon, B. / Haugen, M. (1987): Latin America - Debt Conversion Proliferates, in: Business America, $10 / 13,22.6 .1987$

Hurtienne, T. (1985): Wirtschaftskrise, internationale Verschuldung und Enwicklungspotentiale in Lateinamerika, in: Prokla 59

Johnson, C. (1985): Fleshing Out the Baker Plan for Third World Debt, in: The Banker, 135/718, Dez.

Lever, H. u.a. (1984): The Debt Crisis and the World Economy. Report by a Comonwealth Group of Experts, London

Nunnenkamp, P. / Junge, G. (1985): Die Kreditbeziehungen zwischen westlichen Geschäftsbanken und Entwicklungsländern, Köln

OECD (1987): Financing and External Debt of Developing Countries, Paris

Robert, D. / Remolona, E. (The Group of Thirty) (1987): Finance for Developing Countries, London

Sachs, J. (1986): Managing the LDC Debt Crisis, in: Brookings Papers on Economic Activity, 1

Schatan, J. (1987): World Debt: who is to pay?, London, New Jersey

Schubert, M. (1987): Trading Debt for Equity, in: The Banker, 137/732, März

Schui, H. (1988): Die Schuldenfalle. Schuldenkrise und Dritte-Welt-Politik der USA, Köln

Traber, U. (1986): Neue Formen des Geldes, in: Prokla 63

United Nations (1986): World Economic Survey 1987, Current trends and policies in the world economy, New York, E /1986/59/ST/ESA/183

United States Congress, Joint Economic Committee (1984): International Debt, Hearing before the Subcommittee on Economic Goals and Intergovernmental Policy, Washington, D.C.

Watkins, A. (1986): Till Debt Do Us Part. Who wins, who loses, and who pays for the international debt crisis, Lanham, N.Y., London

Watson, M. / Kincaid, R. u.a. (IMF) (1986): International Capital Markets: developments and prospects, IMF World Economic and Financial Surveys, Washington, D.C.

The World Bank (1987): World Debt Tables. External debt of developing countries, 1986-87, edition, Washington, D.C. 